\title{
A Neutrosophic Approach Based on TOPSIS Method to Image Segmentation
}

\author{
G. Xu, S. Wang, T. Yang, W. Jiang
}

\section{Guojing $\mathrm{Xu}$}

1. Science and Technology on Avionics Integration Laboratory, Shanghai 200233, China

2. China National Aeronautical Radio Electronics Research Institute, Shanghai 200233, China

\section{Shiyu Wang, Tian Yang, Wen Jiang*}

School of Electronics and Information, Northwestern Polytechnical University

Xi'an, Shaanxi Province, 710072, China

*Corresponding author: jiangwen@nwpu.edu.cn, jiangwenpaper@hotmail.com

\begin{abstract}
Neutrosophic set (NS) is a formal framework proposed recently. NS can not only describe the incomplete information in the decision-making system but also depict the uncertainty and inconsistency, so it has applied successfully in several fields such as risk assessment, fuzzy decision and image segmentation. In this paper, a new neutrosophic approach based on TOPSIS method, which can make full use of NS information, is proposed to separate the graphics. Firstly, the image is transformed into the NS domain. Then, two operations, a modified $\alpha$-mean and the $\beta$-enhancement operations are used to enhance image edges and to reduce uncertainty. At last, the segmentation is achieved by the TOPSIS method and the modified fuzzy c-means $(\mathrm{FCM})$. Simulated images and real images are illustrated that the proposed method is more effective and accurate in image segmentation.
\end{abstract}

Keywords: uncertainty; neutrosophic set; TOPSIS method; FCM; image segmentation.

\section{Introduction}

Recently, interests have been growing in the synergistic use of multimodal imaging to get better insights into phenomena or objects. Therefore, the image analysis and processing has become more and more important $[10,22,27,57,66]$. In the existing image processing technology, image segmentation is one of the key tasks in many methods $[2,9,46]$ and computer vision applications $[13,14,21,35]$. The purpose of image segmentation is to divide images into different regions for future processing based on the given criteria. Detecting generic object categories is a fundamental issue in computer vision $[23,36,50,67]$. Its core content is to extract and separate the image information from lots of hurdles like noise, nonuniform illumination, and uneven contrast in otherwise homogenous region [8,31]. For example, in the field of medicine, the complexity of breast cell histopathology $(\mathrm{BCH})$ images makes the reliable segmentation and classification of images quite difficult [20,53]. Some uncertain information in the images, which is not useful for image segmentation, should be dealt properly or even discarded. In practical applications, getting the completely precise information is sometimes impossible $[11,26,34,56,58]$. Therefore, how to deal with the uncertain information effectively is still an open issue. There are already some research about it, for example, information fusion techniques is able to decrease the information's imprecision and uncertainty through the redundancy and complementarity, thereby improve the decision accuracy.

To handle the uncertain information, many mathematical tools are presented such as D-S theory $[6,25,28,48]$, neutrosophy theory [45,64], Z-numbers [5,29,62], single valued neutrosophic cross-entropy $[7,24,54,61]$ and D-numbers [12]. D-S theory was introduced by Dempster and then developed by Shafer, which can well describe and process uncertainty information by assigning 
probability to a set not an element, so it has been widely used in many fields of information processing and fusion.

Neutrosophic set (NS) method reduces the uncertainty information in the image and obtains the better image segmentation results. In neutrosophy set theory, an event $A$ is described by three attributes $\langle A\rangle,\langle A n t i-A\rangle$ and $\langle N e u-A\rangle$, represents the support, opposition and neutrality of $A$, respectively. The neutrality $\langle N e u-A\rangle$ is neither $\langle A\rangle$ nor $\langle A n t i-A\rangle$, which denotes the uncertainty of evidence, in other words, the unknown part of the information. In many cases, the boundaries between these above concepts are vague and may even be crossed together. Recently, Ali proposed the complex neutrosophic set [1]. Wang .et al extended NS to interval neutrosophic sets (INSs) [51] and single-valued neutrosophic sets (SVNSs) [52] which enriched the NS theory and promoted its development. For example, neutrosophy theory has been applied to multi-criteria decision-making $[15,59,60]$. In addition, there are many researches combine NS to image segmentation. Guo et al. $[17,19]$ have applied the NS and clustering method to segment image. Zhang et al. [65] use NS based on watershed method. Some other researchers combine NS with some other method $[39,49,55,63,68]$ to handle image segmentation.

However, some methods do not consider make full use of NS information to segment image. In this paper, a new method with TOPSIS method $[32,37,44]$ is proposed to obtain a better image segmentation result. Firstly, the image is transformed into the NS domain. Secondly, two operations, a modified $\alpha$-mean and $\beta$-enhancement operations are used to reduce the set indeterminacy enhance the image edges. Finally, the segmentation is achieved by the TOPSIS method and the modified fuzzy c-means (FCM).

The remainder of this paper is organized as follows. The basic concepts are briefly introduced in Section 2. In Section 3, the new method is proposed. The simulated images with noise segmentation and real images segmentation are illustrated in Section 4, which show the efficiency of the proposed method. Finally, this paper is concluded in Section 5.

\section{Fuzzy clustering by FCM}

The FCM algorithm makes use of fuzzy membership function which is used to assign a degree of membership for each class $[33,38,43]$. The analysis of FCM algorithm can be defined as the process of organizing objects into groups whose members are similar in some ways [18]. The FCM algorithm belongs to an unsupervised fuzzy clustering algorithm.

Under the condition of a general formulation, the data to be classified can be expressed with a vector $X=\left\{x_{1}, x_{2}, \ldots, x_{M}\right\}$. The vector represents a finite data set of dimension $M$. Assuming that $C>2$ is an integer designating the number of clusters, which sorts the vector $X$. Let $R^{C \times M}$ be the set of all real $C \times M$ matrices. A fuzzy C-partition of $X$ is represented by a matrix

$$
U=\left[\mu_{i k}\right] \in R^{C \times M},
$$

where $\mu_{i k}=\mu_{i}\left(x_{k}\right)$ is the degree of membership of the element $x_{k}$ in the cluster $i$. The entries of the partition matrix come in the form $U$. The constraints following can be verified $[4,40]$ :

$$
\begin{cases}\mu_{i k} \in[0,1], & 1 \leq i \leq C, \quad 1 \leqslant k \leqslant M \\ \sum_{i=1}^{C} \mu_{i k}=1, & 1 \leqslant k \leqslant M \\ \sum_{k=1}^{M} \mu_{i k}>0, & 1 \leqslant i \leqslant C\end{cases}
$$

where $U$ is used to depict the clusters of $X$, and a partition $U$ of $X$ can be obtained by the 
minimization of the FCM objective functional [3]:

$$
J_{q}(U, V: X)=\sum_{k=1}^{M} \sum_{i=1}^{C}\left(\mu_{i k}\right)^{q}\left\|x_{k}-v_{i}\right\|_{A}^{2}
$$

where $q \in[1,+\infty)$ decides the the fuzzy degree of classification results [30], and $V=\left(v_{1}, v_{2}, \ldots, v_{C}\right)$ is the vector of the cluster centers. $\left\|x_{k}-v_{i}\right\|$ is the distance between the gray level of the $\mathrm{k}$-th pixel and the center of the $\mathrm{i}$-th cluster. It is obvious that $\|x\|_{A}=\sqrt{x^{T} A x}$ is any inner product norm where $A$ is any positive definite matrix. The approximate optimization of $J_{q}$ by the FCM algorithm can be obtained by iteration with the following necessary conditions for its local extreme value.

In FCM algorithm $[4,40]$ : assume $\left\|x_{k}-v_{i}\right\|_{A}^{2}>0,1 \leq i \leq C, 1 \leq k \leq M . \quad J_{q}$ can be minimized by $(U, V)$, only if $[4,40]$ as follows:

$$
\begin{gathered}
\mu_{i k}=\left[\sum_{j=1}^{C}\left(\frac{\left\|x_{k}-v_{i}\right\|_{A}}{\left\|x_{k}-v_{j}\right\|_{A}}\right)^{\frac{2}{q-1}}\right]^{-1} \\
v_{i}=\frac{\sum_{k=1}^{M}\left(\mu_{\left.i k^{q} \times x_{k}\right)}\right.}{\sum_{k=1}^{M} \mu_{i k}{ }^{q}}
\end{gathered}
$$

The FCM algorithm is made up of iterations alternating between Eq. (4) and Eq. (5). The algorithm is restrained to either a saddle point of $J_{q}$ or a local minimum.

An algorithm of fast version of the FCM can be applied to an image, which is segmented is depending on a $1-D$ attribute. For example, the gray level. The histogram of image of $L$ levels is represented as $H S$, where $L$ is the number of gray levels. Each pixel of the image has a characteristic which lies in the discrete set.

$$
X=\{0,1, \ldots, L-1\}
$$

In the circumstances, a gray level value is represented by each element of the data set. Then $\mu_{i l}=\mu_{i}(l)$ is used to express the degree of membership of the element $l$ to cluster $i$. The following functional is minimized by the FCM and it is similar to that of Bezdek [3]:

$$
J_{q}(U, V: L)=\sum_{l=0}^{L-1} \sum_{i=1}^{C}\left(\mu_{i l}\right)^{q} \cdot H S(l) \cdot\left\|l-v_{i}\right\|_{A}^{2}
$$

where $\mu_{i l}$ shows the membership degree of the gray level $l$ to cluster $i$.

The FCM is faster than the conventional version processing the whole data set, because that the FCM operates only on the histogram [4]. Where $H S$ be the histogram of image of $L$ levels, $L$ is the number of gray levels, $H S(l)$ is the number of occurrences of the level $l$. The computation of membership degrees of $H S(l)$ pixels is decreased to that of only one pixel with $l$ as gray-level value. General steps of the algorithm are shown as follows in Fig. 1.

In Fig. 1, number of clusters $C$ and the threshold value $\varepsilon$ should be fixed firstly, the $C$ is restricted to a range, where $2<C<L, l=0,1,2, \ldots, L-1$. Gain the $L$ gray levels to initialize the membership degrees $\mu_{i l}$ as follows:

$$
\sum_{i=1}^{C} \mu_{i l}=1, l=0,1, \ldots, L-1 .
$$




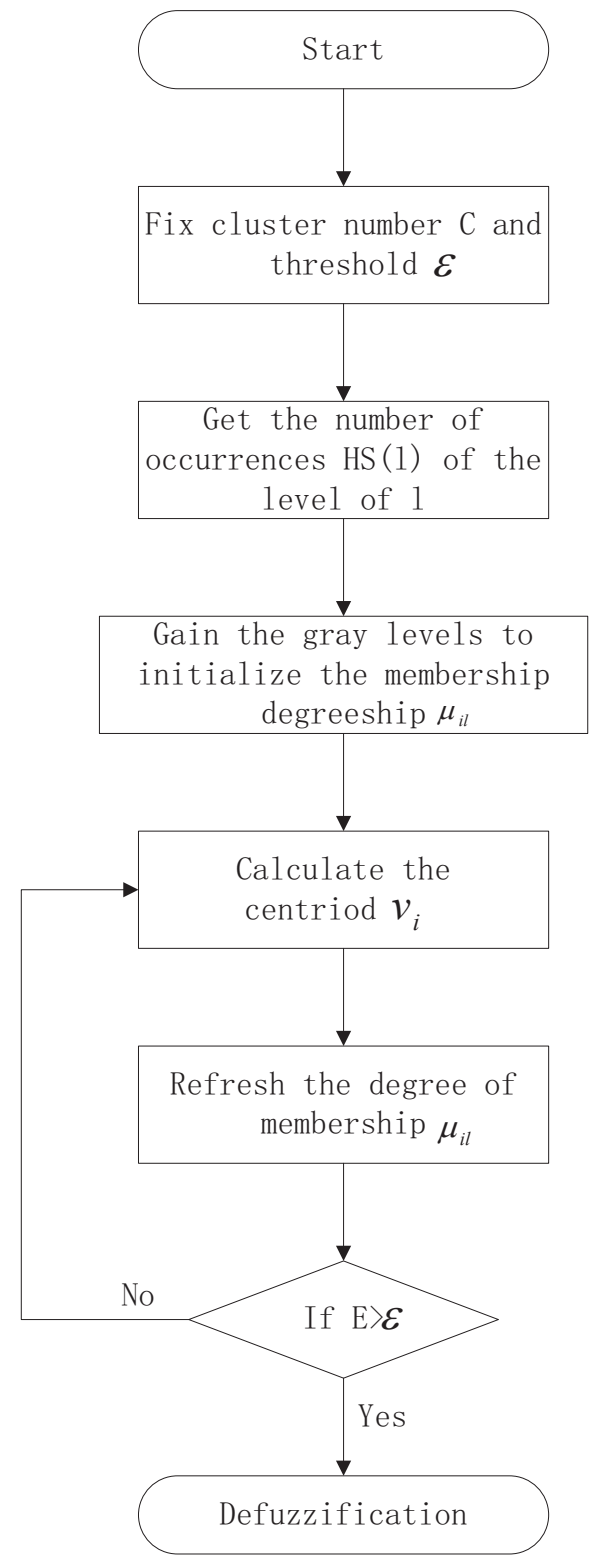

Figure 1: FCM algorithm flow diagram

Centroid $v_{i}$ is calculated like this:

$$
v_{i}=\frac{\sum_{l=0}^{L-1}\left[\mu_{i l}^{q} \cdot H S(l) \cdot l\right]}{\sum_{l=0}^{L-1}\left[\mu_{i l}^{q} \cdot H S(l)\right]}, i=1, \ldots, C
$$

Then refresh the membership degrees as follows: 


$$
\widetilde{\mu}_{i l}=\left[\sum_{j=1}^{C}\left(\frac{\left|l-v_{i}\right|_{A}}{\left|l-v_{j}\right|_{A}}\right)^{\frac{2}{q-1}}\right]^{-1}
$$

The defect measure is presented with $E$, where $E=\sum_{i=1}^{C} \sum_{l=0}^{L-1}\left|\widetilde{\mu}_{i l}-\mu_{i l}\right|$, and then estimate it as follows: If $(E>\varepsilon)\left\{\mu_{i l} \longleftarrow \widetilde{\mu}_{i l}\right\}$.

Finally defuzzification. In this case, the value of clusters is fixed at some exact number, and the value of $q$ is set to 2 . The clustering operation is terminated when $\varepsilon \leq 10^{-3}$, which presents the quality of the clustering results [47].

\section{The proposed method}

The general steps of the proposed method are shown in Fig. 2. The proposed method is achieved by using the modified $\alpha$-mean operation, TOPSIS method $[41,42]$ and the modified FCM. A modified $\alpha$-mean is used to enhance image edges and reduce uncertainty. The $\beta$ enhancement operation is used to enhance the set $T$, which is suitable for segmentation. The TOPSIS method is used to take full advantage of the NS information.

\subsection{Transform the image into NS}

Definition 1 (Neutrosophic image). Let $U$ be a universe of the discourse, and $W \subseteq U$ which is composed by the bright pixels. A neutrosophic image $P_{N S}$ is characterized by three membership sets $T, I$ and $F$ [17].

A pixel $P$ in the given image is described as the neutrosophic set $P(t, i, f)$, which indicates that the pixel $P$ belongs to $W$ in the following way: the probability of $t$ is true, probability of $i$ is indeterminate, and $f$ is false.

The pixel $P(i, j)$ in the image domain is transformed into the neutrosophic domain as follows,

$$
P_{N S}(i, j) \rightarrow\{T(i, j), I(i, j), F(i, j)\}
$$

where $T(i, j), I(i, j)$ and $F(i, j)$ are the membership values. They are calculated by the following equations,

$$
\begin{gathered}
T(i, j)=\frac{\bar{g}(i, j)-\bar{g}_{\text {min }}}{\bar{g}_{\text {max }}-\bar{g}_{\text {min }}}, \\
F(i, j)=\frac{\bar{g}_{\text {max }}-\bar{g}(i, j)}{\bar{g}_{\text {max }}-\bar{g}_{\text {min }}}=1-T(i, j),
\end{gathered}
$$

where

$$
\bar{g}(i, j)=\frac{1}{w \times w} \sum_{m=i-w / 2}^{i+w / 2} \sum_{n=j-w / 2}^{j+w / 2} g(m, n),
$$

and $\bar{g}_{\max }$ and $\bar{g}_{\min }$ are the maximum and minimum of all $\bar{g}(i, j)$, respectively. The indefinite degree is obtained by

$$
I(i, j)=\frac{\delta(i, j)-\delta_{\min }}{\delta_{\max }-\delta_{\min }}
$$

where

$$
\delta(i, j)=|g(i, j)-\bar{g}(i, j)|
$$




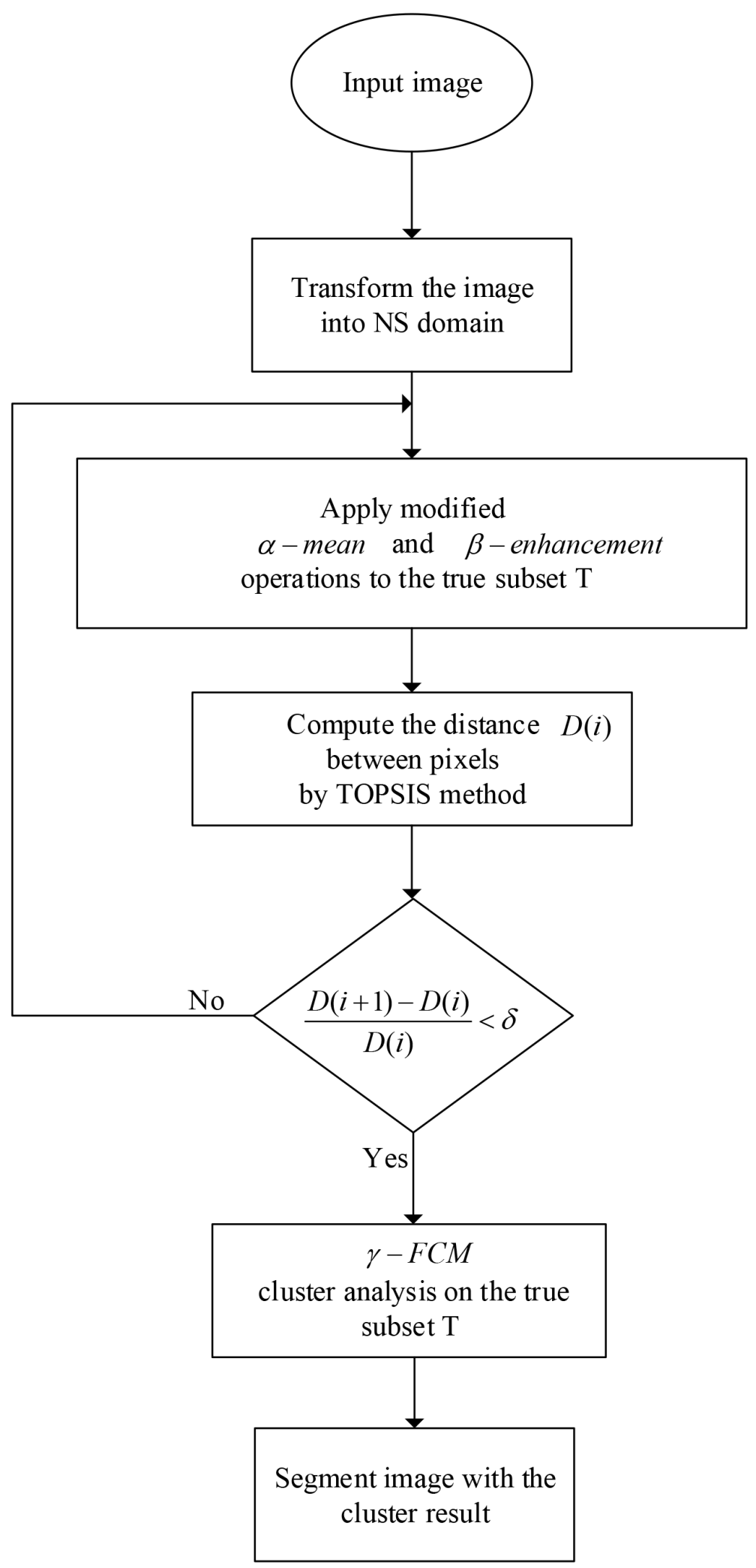

Figure 2: The proposed method 


\subsection{Take modified $\alpha$-mean operation on the true subset $\mathbf{T}$}

Definition 2 ( $\alpha$-mean operation). The modified $\alpha$-mean operation for $P_{N S}, \bar{P}_{N S}(\alpha)$ is

$$
\bar{P}_{N S}(\alpha)=P(T(\alpha), I(\alpha), F(\alpha)) .
$$

The $\alpha$-mean operation of the neutrosophic set is defined as follows,

$$
\begin{gathered}
\bar{T}(\alpha)=\left\{\begin{array}{l}
T, I<\alpha \\
T_{\alpha}^{m}, I \geqslant \alpha
\end{array}\right. \\
T_{\alpha}^{m}(i, j)=\operatorname{Median}(T(m, n)), \\
i-\frac{w}{2} \leqslant m \leqslant i+\frac{w}{2}, j-\frac{w}{2} \leqslant n \leqslant j+\frac{w}{2}
\end{gathered}
$$

where $T_{\alpha}^{m}(i, j)$ is the median of $T(m, n)$,

$$
\begin{gathered}
\bar{F}(\alpha)=1-\bar{T}(\alpha) \\
\overline{I_{\alpha}}(i, j)=\frac{\overline{\delta_{T}}(i, j)-\delta_{T \text { min }}}{\delta_{T \max }-\delta_{T \text { min }}} \\
\overline{\delta_{T}}(i, j)=|\bar{T}(i, j)-\overline{\bar{T}}(i, j)| \\
\overline{\bar{T}}(i, j)=\operatorname{Median}(\bar{T}(m, n)), \\
i-\frac{w}{2} \leqslant m \leqslant i+\frac{w}{2}, j-\frac{w}{2} \leqslant n \leqslant j+\frac{w}{2}
\end{gathered}
$$

After the modified $\alpha$-mean operation, the distribution of the elements in $I$ becomes more uniform. Furthermore, the image edges are enhanced, and the membership set $\mathrm{T}$ becomes more distinct, which is suitable for segmentation. In this paper, $\alpha$ is 0.85 according to the experimental results.

\subsection{Take $\beta$-enhancement operation on the true subset $\mathbf{T}$}

The intensification operation for the membership $\mu$ in fuzzy set is defined as follows

$$
\begin{aligned}
\mu^{\prime}(i, j) & =f(\mu(i, j)) \\
& =\left\{\begin{array}{l}
2 \mu^{2}(i, j), \mu(i, j) \leqslant 0.5 \\
1-2(1-\mu(i, j))^{2}, \mu(i, j)>0.5
\end{array}\right.
\end{aligned}
$$

Definition 3 ( $\beta$-enhancement operation). A $\beta$-enhancement operation for $P_{N S}, P_{N S}^{\prime}(\beta)$, is defined as follows [17]

$$
\begin{gathered}
P_{N S}^{\prime}(\beta)=P\left(T^{\prime}(\beta), I^{\prime}(\beta), F^{\prime}(\beta)\right) \\
T^{\prime}(\beta)=\left\{\begin{array}{l}
T, I<\beta \\
T_{\beta}^{\prime}, I \geqslant \beta
\end{array}\right. \\
F^{\prime}(\beta)=\left\{\begin{array}{l}
F, I<\beta \\
F_{\beta}^{\prime}, I \geqslant \beta
\end{array}\right.
\end{gathered}
$$

where $\beta=\overline{I(i, j)}$ is a dynamic parameter that adjusts the threshold based on the image.

$$
T_{\beta}^{\prime}(i, j)=\left\{\begin{array}{l}
2 T^{2}(i, j), T(i, j) \leqslant 0.5 \\
1-2(1-T(i, j))^{2}, T(i, j)>0.5
\end{array}\right.
$$




$$
\begin{gathered}
F_{\beta}^{\prime}(i, j)=\left\{\begin{array}{l}
2 F^{2}(i, j), F(i, j) \leqslant 0.5 \\
1-2(1-F(i, j))^{2}, F(i, j)>0.5
\end{array}\right. \\
I_{\beta}^{\prime}(i, j)=\frac{\delta_{T}^{\prime}(i, j)-\delta_{T \min }^{\prime}}{\delta_{T \max }^{\prime}-\delta_{T \min }^{\prime}} \\
\delta_{T}^{\prime}(i, j)=\left|T^{\prime}(i, j)-\bar{T}^{\prime}(i, j)\right| \\
\bar{T}^{\prime}(i, j)=\frac{1}{w \times w} \sum_{m=i-w / 2}^{i+w / 2} \sum_{n=j-w / 2}^{j+w / 2} T^{\prime}(m, n) .
\end{gathered}
$$

After the $\beta$-enhancement operation, the membership set $T$ becomes more distinct and has less information redundancy, which is appropriate for segmentation.

\subsection{TOPSIS method based on NS}

Definition 4. Assume that an completely accurate pixel $P_{N S t u r e}(i, j)=[1,0,0]$, in the image is described that it belongs to $\mathrm{W}$ without any doubt. Moreover, $P_{N S f a l s e}(i, j)=[0,1,1]$ is described that it does not belongs to W. $D(i)$ in the image indicates the distance between the pixel $P_{N S}(i, j)$ and pix $P_{N \text { Sture }}(i, j)$

$$
\begin{aligned}
D(i) & =\frac{D\left(P_{\text {NS }}(i, j), P_{\text {NSture }}(i, j)\right)}{D\left(P_{\text {NSture }}(i, j), P_{\text {NSfalse }}(i, j)\right)} \\
& =\frac{\sqrt{(T(i)-1)^{2}+(I(i)-0)^{2}+(F(i)-0)^{2}}}{\sqrt{(1-0)^{2}+(0-1)^{2}+(0-1)^{2}}}
\end{aligned}
$$

In the method, the information of the pixels are made full use. In addition to the probability $T$ and $F$ that the pixels is in a category or not, its degree of neutrality $I$ is also considered. In other words, uncertain information in the obtained image is involved in the clustering process. Accordingly, our method is more accurate and more reasonable in image segmentation.

\subsection{Modified FCM based on NS}

Considering the effect of indeterminacy, two sets, $T$ and $I$ are composed into a new value for FCM clustering:

$$
X(i, j)=\left\{\begin{array}{l}
T(i, j), I(i, j) \leqslant \gamma \\
\overline{T_{\gamma}}(i, j), I(i, j)>\gamma
\end{array}\right.
$$

Here, $\gamma=0.85$ is determined by experiments.

Then the modified FCM is applied to the subset $T$ as introduced in Section 2. First, the pixel information in the matrix $X(i, j)$ is transformed into a vector $x$, then the image modified information vector $x$ is subjected to the following recursive algorithm for cluster segmentation.

$$
\begin{gathered}
J_{q}(U, V: X)=\sum_{k=1}^{M} \sum_{i=1}^{C}\left(\mu_{i k}\right)^{q}\left\|x_{k}-v_{i}\right\|_{A}^{2} \\
\mu_{i k}=\left[\sum_{j=1}^{C}\left(\frac{\left\|x_{k}-v_{i}\right\|_{A}}{\left\|x_{k}-v_{j}\right\|_{A}}\right)^{\frac{2}{q-1}}\right]^{-1}
\end{gathered}
$$




$$
v_{i}=\frac{\sum_{k=1}^{M}\left(\mu_{i k}^{q} \times x_{k}\right)}{\sum_{k=1}^{M} \mu_{i k}{ }^{q}}
$$

where $J_{q}(U, V: X)$ can be minimized by $(U, V)$.

The proposed method can be summarized as the following steps:

Step 1. Transform the image into NS domain using Eq. (12) to Eq. (16).

Step 2. Perform the modified $\alpha$-mean and $\beta$-enhancement operations on the subset $T$ through Eq. (17) to Eq. (32).

Step 3. Compute the distance $D(i)$ using Eq. (33).

Step 4. If $(D(i+1)-D(i)) / D(i)<\delta$, go to Step 5; else go back to Step 2.

Step 5. Apply the modified FCM to the subset $T$ by Eq. (34) to Eq. (37).

Step 6. Segment image.

\section{Experiments and discussions}

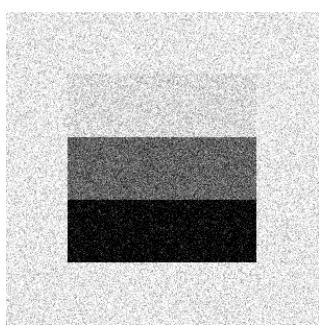

(a)

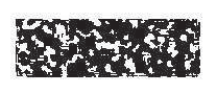

(b)

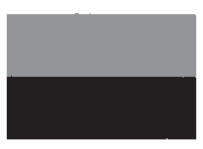

(c)

Figure 3: Simulated image 1 used for segmentation: (a) original simulated image, (b) segmentation result of Guo's method [17], (c) segmentation result of proposed method

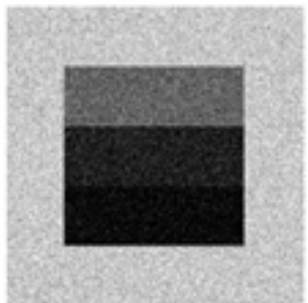

(a)

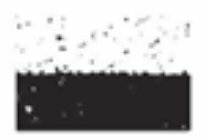

(b)

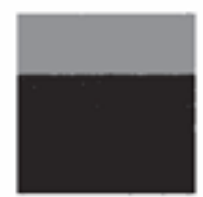

(c)

Figure 4: Simulated image 2 used for segmentation:(a) original simulated image, (b) segmentation result of Guo's method [17], (c) segmentation result of proposed method

In order to test the effectiveness of the proposed method, the Gaussian noise whose expectation is 0 and the variance is 0.015 , that is, $N(0,0.015)$ is added to two simulated images. As 
shown in Fig. 3(a), the upper gray belt is close to the background gray, which is easy to be confused. In Fig. 4(a), gray belts of two layers below are difficult to be distinguished. Fig. 3(b) and Fig. 4(b) are the segmentation results of the Guo's method [17]. In Fig. 3(c) and Fig. $4(\mathrm{c})$, segmentation results of the proposed method in this paper, the shape and size of image are segmented better. The three regions are distinct and easy to be recognized by the proposed approach. The edges of the image are also more clear. The error segmentation rates of Fig. 3(b) and Fig. 4(b) are 0.424 and 0.611 , which show that many pixels are wrongly segmented by the Guo's method [17]. The error segmentation rates of Fig. 3(c) and Fig. 4(c) are 0.243 and 0.384, which perform that a less of pixels are wrongly segmented by the proposed method.

Then let's consider the consequent of dividing some actual images. Fig. 5(a) and Fig. 6(a) are real lena images with different noise levers. The error segmentation rates are 0.815 and 0.718 in Fig. 5(b) and Fig. 6(b) segmented by Guo, respectively. However, the false judgement rate of our proposed method is just 0.190 and 0.367 in Fig. 5(c) and Fig. 6(c). In the split photos, hat, hair and woman's face, three main areas in the image are segmented more correctly and clearly. More pixels are segmented correctly and the regions are more consistent and homogenous in Fig. 5(c) and Fig. 6(c), which are better to be distinguished.

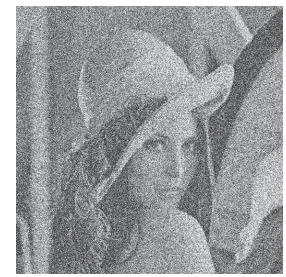

(a)

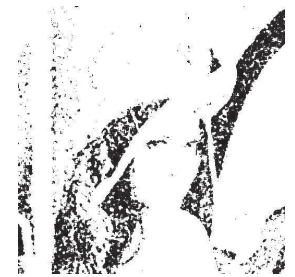

(b)

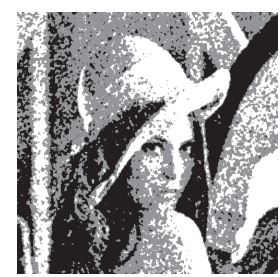

(c)

Figure 5: Real image used for segmentation: (a) original Lena image with gaussian noise; (b) segmentation result of Guo's method [17]; (c) segmentation result of proposed method

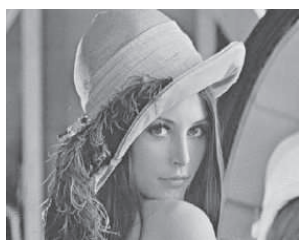

(a)

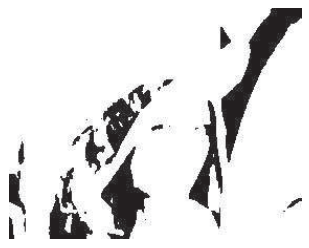

(b)

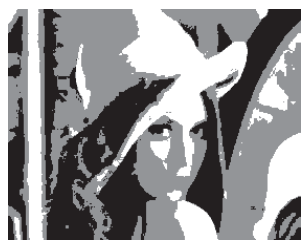

(c)

Figure 6: Real image used for segmentation: (a) original Lena image; (b) segmentation result of Guo's method [17]; (c) segmentation result of proposed method

\section{Conclusions}

In this paper, a new method with the TOPSIS method, which effectively enhances the image edges and reduces the indeterminacy with the modified $\alpha$-mean operation and $\beta$-enhancement operation, is proposed. With the TOPSIS method, the NS information in the image is fully utilized. The information of $T, I$ and $F$ in the NS domain is made the best. The experimental results with simulated images and real images show that the proposed method is more effective in handling vague edges and in better making full use of NS information. With different noise levels and different kinds of noise, especially complex noise contained in the real medical images, 
the proposed method performs better and more accurately than the existing method. Image segmentation results are obtained more precisely and reasonably. At the same time, the proposed method shows that the notable validity in increasing boundary accuracy and region harmony. The comparison between the experimental results and the error segmentation rates can illustrate that our proposed method can handle the uncertain and indeterminate information better. In the further research directions, it may find more applications in diverse fields of control theory, computer vision, and artificial intelligence, especially in the image processing field.

\section{Acknowledgments}

The work is partially supported by Science and Technology on Avionics Integration Laboratory and Aviation Science Foundation (Program No. 20165553036).

\section{Conflict of interests}

The authors declare that there is no conflict of interests regarding the publication of this paper.

\section{Bibliography}

[1] Ali, M.; Smarandache, F. (2017); Complex neutrosophic set, Neural Computing \& Applications, 28(7), 1817-1834, 2017.

[2] Ashraf, M.; Sarim, M.; Shaikh, A.B. (2017). Cellular-Cut-Interactive n-Dimensional Image Segmentation Using Cellular Automata. International Journal of Pattern Recognition \& Artificial Intelligence, 2017.

[3] Bezdek, J.C.; Ehrlich, R.; Full, W. (1984). FCM: The fuzzy c-means clustering algorithm, Computers \& Geosciences, 10(2-3), 191-203, 1984.

[4] Cannon, R.L.; Dave, J.V.; Bezdek, J.C. (1986). Efficient Implementation of the Fuzzy c-Means Clustering Algorithms, IEEE Transactions on Pattern Analysis \& Machine Intelligence, 8(2), 248-255, 1986.

[5] Chen, L.; Deng, Y. (2018). A new failure mode and effects analysis model using DempsterShafer evidence theory and grey relational projection method, Engineering Applications of Artificial Intelligence, 76, 13-20, 2018, doi:10.1016/j.engappai.2018.08.010.

[6] Dempster, A.P. (1967). Upper and Lower Probabilities Induced by a Multi-valued Mapping, Annals of Mathematical Statistics, 38(2),325-339, 1967.

[7] Deng, X. (2018). Analyzing the monotonicity of belief interval based uncertainty measures in belief function theory, International Journal of Intelligent Systems, 33(9),1869-1879, 2018 .

[8] Deng, X.; Han, D.; Dezert, J.; Deng, Y.; Shyr, Y. (2016). Evidence combination from an evolutionary game theory perspective, IEEE Transactions on Cybernetics, 46(9), 2070-2082, 2016.

[9] Deng, X. Jiang, W. (2018). Dependence assessment in human reliability analysis using an evidential network approach extended by belief rules and uncertainty measures, Annals of Nuclear Energy, 117, 183-193, 2018. 
[10] Deng, X.; Jiang, W.; Wang, Z. (2019). Zero-sum polymatrix games with link uncertainty: A Dempster-Shafer theory solution, Applied Mathematics and Computation, 340, 101-112, 2019 .

[11] Deng X.; Xiao, F.; Deng, Y. (2017). An improved distance-based total uncertainty measure in belief function theory, Applied Intelligence, 46(4), 898-915, 2017.

[12] Deng, Y. (2012). D numbers: theory and applications, Journal of Information \& Computational Science, 9(9), 2421-2428, 2012.

[13] Dou, R.; Nan, G. (2018). Optimizing sensor network coverage and regional connectivity in industrial IoT systems, IEEE Systems Journal, 11(3), 1351-1360, 2018.

[14] Eklund, A.; Dufort, P.; Forsberg, D.; Laconte, S.M. (2013). Medical image processing on the GPU: Past, present and future, Medical Image Analysis, 17(17), 1073-1094, 2013.

[15] Fei, L.; Deng, Y.; Hu, Y. (2018). DS-VIKOR: A New Multi-criteria Decision-Making Method for Supplier Selection, International Journal of Fuzzy Systems, 2018, p. 10.1007/s40815$018-0543-\mathrm{y}$.

[16] Greco, S.; Matarazzo, B.; Slowinski, R. (2001). Rough sets theory for multicriteria decision analysis, European Journal of Operational Research, 129(1), 1-47, 2001.

[17] Guo, Y.; Cheng, H.D. (2009). New neutrosophic approach to image segmentation, Pattern Recognition, 42(5), 587-595, 2009.

[18] Guo, Y.; Sengur, A. (2015). NECM: Neutrosophic evidential c -means clustering algorithm, Neural Computing \& Applications, 26(3), 561-571, 2015.

[19] Guo, Y.; Sengur, A. (2013). A novel color image segmentation approach based on neutrosophic set and modified fuzzy c -means, Circuits, Systems, and Signal Processing, 32(4), 1699-1723, 2013.

[20] Han, Y.; Deng, Y. (2018). An enhanced fuzzy evidential DEMATEL method with its application to identify critical success factors, Soft computing, 22(15), 5073-5090, 2018.

[21] He, Z.; Jiang, W. (2018). An evidential Markov decision making model, Information Sciences, 467, 357-372, 2018.

[22] He, Z.; Jiang, W. (2018). An evidential dynamical model to predict the interference effect of categorization on decision making, Knowledge-Based Systems, 150, 139-149, 2018.

[23] Hong, C.; Zhang, J.; Cao, X.B.; Du, W.B. (2016). Structural properties of the Chinese air transportation multilayer network, CHAOS SOLITONS \& FRACTALS, 86, 28-34, 2016.

[24] Hu, K.; Ye, J.; Fan, E.; Shen, S.; Huang, L.; Pi, J. (2017). A novel object tracking algorithm by fusing color and depth information based on single valued neutrosophic cross-entropy, Journal of Intelligent \& Fuzzy Systems, 32(3), 1775-1786, 2017.

[25] Jiang, W. (2018). A correlation coefficient for belief functions, International Journal of Approximate Reasoning, 2018, p. Published on line, Doi: 10.1016/j.ijar.2018.09.001.

[26] Jiang, W.; Hu, W. (2018). An improved soft likelihood function for Dempster-Shafer belief structures, International Journal of Intelligent Systems, 33(6), 1264-1282, 2018. 
[27] Jiang, W.; Huang, C. (2018). A Multi-criteria Decision-making Model for Evaluating Suppliers in Green SCM, International Journal of Computers Communications \& Control, 13(3), 337-352, 2018.

[28] Jiang, W.; Wang, S. (2017). An Uncertainty Measure for Interval-valued Evidences, International Journal of Computers Communications \& Control, 12(5), 631-644, 2017.

[29] Kang, B.; Deng, Y.; Hewage, K.; Sadiq, R. (2018). A method of measuring uncertainty for Znumber, IEEE Transactions on Fuzzy Systems, 2018, p. DOI:10.1109/TFUZZ.2018.2868496.

[30] Kannan, S.R.; Ramathilagam, S.; Devi R.; Hines, E. (2012). Strong fuzzy c-means in medical image data analysis, Journal of Systems and Software, 85(11), 2425-2438, 2012, doi:10.1016/j.jss.2011.12.020.

[31] Kittaneh, O.A.; Khan, M.A.U.; Akbar, M.; Bayoud H.A. (2016). Average Entropy: A New Uncertainty Measure with Application to Image Segmentation, American Statistician, 70, 18-24, 2016.

[32] Kuo, T. (2016). A modified TOPSIS with a different ranking index, European Journal of Operational Research, 260, 2016.

[33] Li, P.; Chen, Z.; Yang, L.T.; Zhao, L.; Zhang, Q. (2017). A privacy-preserving high-order neuro-fuzzy c-means algorithm with cloud computing, Neurocomputing, 2017.

[34] Li, Y.; Deng, Y. (2018). Generalized Ordered Propositions Fusion Based on Belief Entropy, International Journal of Computers Communications \& Control, 13(5), 792-807, 2018.

[35] Li, Z.; Chen, L.; Nan, G. (2018). Small-scale Renewable Energy Source Trading: A Contract Theory Approach, IEEE Transactions on Industrial Informatics, 14(4), 1491-1500, 2018.

[36] Liang, W.; He, J.; Wang, S.; Yang, L.; Chen, F. (2018). Improved cluster collaboration algorithm based on wolf pack behavior, Cluster Computing, 2018, p. Published on line, doi: $10.3390 / \mathrm{s} 17040922$.

[37] Lourenzutti, R.; Krohling, R.A.; Reformat, M.Z. (2017). Choquet based TOPSIS and TODIM for dynamic and heterogeneous decision making with criteria interaction, Information Sciences, 408, 41-69, 2017.

[38] Mahela, O.P.; Shaik, A.G. (2017). Power quality recognition in distribution system with solar energy penetration using S -transform and Fuzzy C-means clustering, Renewable Energy, 106, 37-51, 2017.

[39] Mohan, J.; Krishnaveni, V.; Guo, Y. (2013). MRI denoising using nonlocal neutrosophic set approach of wiener filtering, Biomedical Signal Processing \& Control, 8(6), 779-791, 2013.

[40] Muller, H.; Michoux, N.; Bandon, D.; Geissbuhler, A. (2004). A review of content based image retrieval systems in medical applications clinical benefits and future directions, International Journal of Medical Informatics, 73(1), 1-23, 2004.

[41] Nădăban, S.; Dzitac, S. (2016). Neutrosophic TOPSIS: A general view, 2016 6th International Conference on Computers Communications and Control, IEEE, 250-253, 2016.

[42] Nădăban, S.; Dzitac, S.; Dzitac, I. (2016). Fuzzy TOPSIS: A general view, Procedia Computer Science, 91, 823-831, 2016. 
[43] Nayak, J.; Naik, B.; Behera, H.S.; Abraham, A.(2017); Hybrid Chemical Reaction based Metaheuristic with Fuzzy c-means Algorithm for Optimal Cluster Analysis, Expert Systems with Applications, 79, 282-295, 2017.

[44] Onu, U.P.; Xie, Q.; Xu, L. (2017). A Fuzzy TOPSIS model Framework for Ranking Sustainable Water Supply Alternatives, Water Resources Management An International Journal Published for the European Water Resources Association, 1-15, 2017.

[45] Peng, J.; Wang, J.; Wu, X.; Wang, J.; Chen, X. (2015). Multi-valued Neutrosophic Sets and Power Aggregation Operators with Their Applications in Multi-criteria Group Decisionmaking Problems, International Journal of Computational Intelligence Systems, 8(2), 345$363,2015$.

[46] Qian, P.; Zhao, K.; Jiang, Y.; Su, K.H.; Deng, Z.; Wang, S.; et al. (2017). Knowledgeleveraged transfer fuzzy C -Means for texture image segmentation with self-adaptive cluster prototype matching, Knowledge-Based Systems, 2017.

[47] Reyes-Galaviz, O.F.; Pedrycz, W. (2017). Enhancement of The Classification and Reconstruction Performance of Fuzzy C-Means with Refinements of Prototypes, Fuzzy Sets \& Systems, 318, 80-99, 2017.

[48] Shafer, G. (1976). A Mathematical Theory of Evidence, New Jersey, Princetion University Press, 1976.

[49] Shan, J.; Cheng, H.D.; Wang, Y. (2012). A novel segmentation method for breast ultrasound images based on neutrosophic l-means clustering, Medical Physics, 39(9), 5669-5682, 2012.

[50] Wang, B.; Xiong, H.; Jiang, X.; Zheng, Y.F. (2014). Data-Driven Hierarchical Structure Kernel for Multiscale Part-Based Object Recognition, IEEE Transactions on Image Processing, 23(4), 1765-1778, 2014, doi:10.1109/TIP.2014.2307480.

[51] Wang, H.; Smarandache, F.; Sunderraman, R.; Zhang, Y.Q. (2005). Interval Neutrosophic Sets and Logic: Theory and Applications in Computing: Theory and Applications in Computing. vol. 5, Infinite Study, 2005.

[52] Wang, H.; Smarandache, F.; Zhang, Y.; Sunderraman, R. (2010). Single valued neutrosophic sets, Rev Air Force Acad, 17, 4-10, 2010.

[53] Wang, P.; Hu, X.; Li, Y.; Liu, Q.; Zhu, X. (2016). Automatic cell nuclei segmentation and classification of breast cancer histopathology images, Signal Processing 122, 1 - 13, 2016.

[54] Xiao, F. (2019). Multi-sensor data fusion based on the belief divergence measure of evidences and the belief entropy, Information Fusion, 46(2019), 23-32, 2019;.

[55] Xin, Z.; Shitong, W. (2012). Neutrosophic image segmentation approach based on similarity, Application Research of Computers, 29(6), 2371-2374, 2012.

[56] Xiong, H.; Zheng, D.; Zhu, Q.; Wang, B.; Zheng, Y.F. (2013). A Structured Learning-Based Graph Matching Method for Tracking Dynamic Multiple Objects, IEEE Transactions on Circuits and Systems for Video Technology, 23(3), 534-548, 2013, doi:10.1109/TCSVT.2012.2210801.

[57] Xu, S.; Jiang, W.; Deng, X.; Shou, Y. (2018). A modified Physarum-inspired model for the user equilibrium traffic assignment problem, Applied Mathematical Modelling, 55, 340-353, 2018. 
[58] Xu, Z.; Hu, C.H.; Yang, F.; Kuo, S.H.; Goh, C.K.; Gupta, A.; et al. (2017). Data-driven Inter-Turn Short Circuit Fault Detection in Induction Machines, IEEE Access, 5(1), 2505525068, 2017.

[59] Ye, J. (2013). Multicriteria decision-making method using the correlation coefficient under single-valued neutrosophic environment, International Journal of General Systems, 42(4), 386-394, 2013.

[60] Ye, J. (2014). A multicriteria decision-making method using aggregation operators for simplified neutrosophic sets, Journal of Intelligent \& Fuzzy Systems, 26(5), 2459-2466, 2014.

[61] Yin, L.; Deng, Y. (2018). Toward uncertainty of weighted networks: An entropybased model, Physica A: Statistical Mechanics and its Applications, 508, 176-186, 2018, doi:http://doi.org/10.1016/j.physa.2018.05.067.

[62] Zadeh, L.A. (2011). A Note on Z-numbers, Information Sciences, 181(14), 2923-2932, 2011.

[63] Zhang, G.; Wang, D. (2014). Neutrosophic image segmentation approach integrated LPG \& PCA, Journal of Image \& Graphics, 19(5), 693-700, 2014.

[64] Zhang, H.; Ji, P.; Wang, J.; Chen, X. (2015). An Improved Weighted Correlation Coefficient Based on Integrated Weight for Interval Neutrosophic Sets and its Application in Multicriteria Decision-making Problems, International Journal of Computational Intelligence Systems, 8(6), 1027-1043, 2015.

[65] Zhang, M.; Zhang, L.; Cheng, H.D. (2010). A neutrosophic approach to image segmentation based on watershed method, Signal Processing, 90(5), 1510-1517, 2010.

[66] Zhang, X.; Mahadevan, S. (2017). Aircraft re-routing optimization and performance assessment under uncertainty, Decision Support Systems, 96, 67-82, 2017.

[67] Zhang, X.; Mahadevan, S.; Deng, X. (2017). Reliability analysis with linguistic data: An evidential network approach, Reliability Engineering \& System Safety, 162, 111-121, 2017.

[68] Zhao, X.; Wang, S.T.; Juna, W.U. (2011). Neutrosophic image segmentation approach based on thermal balance, Computer Engineering, 37(19), 210-212, 220, 2011. 